Research Article

\title{
Red Ginseng Inhibits Tau Aggregation and Promotes Tau Dissociation In Vitro
}

\author{
Soo Jung Shin, ${ }^{1}$ Yong Ho Park, ${ }^{1}$ Seong Gak Jeon, ${ }^{1}$ Sujin Kim, ${ }^{1}$ Yunkwon Nam, \\ Sang-Muk Oh, ${ }^{1}$ Yong Yook Lee $\mathbb{D}^{2},{ }^{2}$ and Minho Moon ${ }^{1}{ }^{1}$ \\ ${ }^{1}$ Department of Biochemistry, College of Medicine, Konyang University, Daejeon 35365, Republic of Korea \\ ${ }^{2}$ The Korean Ginseng Research Institute, Korea Ginseng Corporation, Gajeong-ro 30, Shinseong-dong, Yuseong-gu, \\ Daejeon 34128, Republic of Korea
}

Correspondence should be addressed to Yong Yook Lee; ace28@kgc.co.kr and Minho Moon; hominmoon@konyang.ac.kr Received 15 April 2020; Revised 21 May 2020; Accepted 28 May 2020; Published 1 July 2020

Guest Editor: Marcos R. de Oliveira

Copyright (c) 2020 Soo Jung Shin et al. This is an open access article distributed under the Creative Commons Attribution License, which permits unrestricted use, distribution, and reproduction in any medium, provided the original work is properly cited.

\begin{abstract}
Tau, a microtubule-associated protein expressed in mature neurons, interacts with tubulin to promote the assembly and stabilization of microtubules. However, abnormally hyperphosphorylated tau dissociates from microtubules and self-aggregates. Tau aggregates, including paired helical filaments and neurofibrillary tangles, promote neuronal dysfunction and death and are the defining neuropathological feature of tauopathies. Therefore, suppressing tau aggregation or stimulating the dissociation of tau aggregates has been proposed as an effective strategy for treating neurodegenerative diseases associated with tau pathology such as Alzheimer's disease (AD) and frontotemporal dementia. Interestingly, ginsenosides extracted from Panax ginseng reduced the hippocampal and cortical expression of phosphorylated tau in a rat model of AD. However, no studies have been conducted into the effect of red ginseng (RG) and its components on tau pathology. Here, we evaluated the effect of Korean red ginseng extract (KRGE) and its components on the aggregation and disassociation of tau. Using the thioflavin $\mathrm{T}$ assay, we monitored the change in fluorescence produced by the aggregation or disassociation of tau K18, an aggregation-prone fragment of $\operatorname{tau}_{441}$ containing the microtubule-binding domain. Our analysis revealed that KRGE not only inhibited tau aggregation but also promoted the dissociation of tau aggregates. In addition, the KRGE fractions, such as saponin, nonsaponin, and nonsaponin fraction with rich polysaccharide, also inhibited tau aggregation and promoted the dissociation of tau aggregates. Our observations suggest that RG could be a potential therapeutic agent for the treatment of neurodegenerative diseases associated with tauopathy.
\end{abstract}

\section{Introduction}

Tau, a microtubule-associated protein expressed in neurons, interacts with tubulin and promotes the assembly and stabilization of microtubules $[1,2]$. Alternative splicing of the MAPT (microtubule-associated protein tau) gene produces six isoforms of tau. These are classified according to the number of repeats of 29 amino acids on the $\mathrm{N}$-terminal region $(\mathrm{N}$ : zero, one, or two) and the number of microtubule-binding domain repeats ( $\mathrm{R}$ : three or four) on the C-terminal region $[3,4]$. The largest tau isoform is $4 \mathrm{R} 2 \mathrm{~N}$ tau, and this isoform is the most effective at promoting microtubule assembly
$[5,6]$. As a microtubule-associated phosphoprotein, the affinity of tau for microtubules is dependent on its phosphorylation level, and normal tau phosphorylation is essential for neuronal plasticity and axonal outgrowth $[7,8]$. However, abnormally hyperphosphorylated tau is released from microtubules due to its reduced biological activity and induces synaptic terminal alteration and axonal degeneration, which can result in cognitive impairment [9]. In addition, tau released from microtubules self-assembles into neurotoxic insoluble aggregates such as paired helical filaments, straight filaments, and neurofibrillary tangles (NFTs) [10]. In particular, NFTs in the brain are a histopathological feature of 
tauopathies such as Alzheimer's disease (AD), frontotemporal dementia, Parkinson's disease, Pick's disease, and progressive supranuclear palsy [11-15].

Abnormally hyperphosphorylated tau inhibits and disrupts the assembly of microtubules [16]. In addition, numerous studies have demonstrated the toxicity of abnormal tau aggregates in neurons and glial cells [16]. While soluble tau is nontoxic, tau aggregates promote the degeneration of N2a neuroblastoma cells [17]. Moreover, tau dimers suppress axonal transport in isolated squid axoplasm [18], and the neurotoxicity of tau trimers was demonstrated in both SH-SY5Y cells and the mouse hippocampal neurons $[19,20]$. Interestingly, several studies have shown that tau oligomers and aggregates can be anterogradely propagated between cells via exosomes, endocytosis, and macropinocytosis both in vitro and in vivo [21-24]. Furthermore, insoluble oligomeric tau has been implicated in the dysfunction of the ubiquitin-proteasome system [25]. Moreover, mice expressing antiaggregation mutations in tau do not exhibit tau-related neuropathology [26], and inhibition of tau aggregation alleviates tauopathy in the C. elegans model of tauopathy and P301S tau transgenic mice [27, 28]. Indeed, clinical trials are currently underway to investigate the efficacy of methylene blue (Texas Alzheimer's Research and Care Consortium), NPT088 (Proclara), and LY3303560 (Lilly), all of which are agents that that can inhibit, dissociate, and neutralize tau aggregation, for the treatment of AD [29]. Thus, inhibition of tau aggregation is a wellestablished therapeutic strategy for the treatment of tauopathies including $\mathrm{AD}$ [30].

Ginseng, the root of Panax ginseng Meyer, is a representative medicinal herb in East Asian countries. Ginseng contains various bioactive components such as ginsenosides, flavonoids, polyphenols, and polysaccharides [31]. Interestingly, ginseng can be processed into red ginseng (RG) through a series of steam and drying processes to enhance the pharmacological efficacy of the bioactive substances present in ginseng [32]. In addition, ginseng has been used as a medicinal herb that can treat various diseases such as cancer, diabetes, and cardiovascular diseases [33-35]. Moreover, RG has been reported to mitigate ADrelated pathology in animal models of $\mathrm{AD}$ and patients with $\mathrm{AD}$ [36-40]. Furthermore, ginsenosides have been shown to ameliorate $\mathrm{AD}$-related pathology, potentially by modulating amyloid- $\beta$ ( $\mathrm{A} \beta$ ) processing, phosphorylated tau ( $\mathrm{p}$-tau) expression, and choline acetyltransferase expression [41, 42]. Interestingly, although the mechanism underlying their effect remains unknown, treatment with ginsenosides extracted from Panax ginseng reduced p-tau deposition in a rat model of $\mathrm{AD}$ [42]. However, to date, the effects of $\mathrm{RG}$ and its components on the aggregation and dissociation of tau have not yet been examined. In this study, we investigated the effect of Korean red ginseng extract (KRGE) and KRGE fractions, including the saponin fraction (SF), nonsaponin fraction (NSF), and nonsaponin fraction with rich polysaccharide (NFP), on tau aggregation and dissociation. To do so, we performed the thioflavin T (ThT) assay with tau K18 fragment, a microtubule-binding domain repeat of the $4 \mathrm{R} 2 \mathrm{~N}$ tau isoform.

\section{Materials and Methods}

2.1. Chemicals and Materials. Commercial KRGE was purchased from the Korea Ginseng Corporation (CheongKwan-Jang, Daejeon, Republic of Korea). KRGE was prepared from 6-year-old Korean Panax ginseng Meyer. Thioflavin T (Basic Yellow 1, ThT) and Dulbecco's phosphate-buffered saline (PBS) were purchased from the Tokyo Chemical Industry Co., Ltd. (Tokyo, Japan; T0558) and Welgene Inc. (Gyeongsan, Republic of Korea; LB 001-02), respectively. Methylene blue (M9140-25G), 1,4-dithiothreitol (DTT, D0632-5G), and heparin sodium salt from porcine intestinal mucosa (H3393-25KU) were purchased from Sigma-Aldrich (St. Louis, MO, USA). Some carbohydrate-digesting enzymes, namely, $\alpha$-amylase from Aspergillus oryzae, amyloglucosidase from Aspergillus niger, and pectinesterase from orange peel, were all obtained from Sigma-Aldrich, whereas polygalacturonase from Aspergillus aculeatus was purchased from Megazyme (Bray, Ireland). A $\beta_{1-42}$ peptide was purchased from Bachem, Inc. (Torrance, CA, USA; H-1366).

2.2. Extraction of KRGE Fractions. In the previous study, the commercial KRGE was freeze-dried and ground into a fine powder [36]. The KRGE for SF, NSF, and NFP (Figure S1) was supplied by the Korea Ginseng Corporation (Buyeo, Republic of Korea) according to the reported method [32]. The SF and NSF were prepared from the KRGE by adsorption chromatography with Diaion HP20 (Mitsubishi Chemical Industries, Ltd.). The KRGE was diluted to $10 \%$ in water and then filtered. The diluted KRGE was subjected to adsorption chromatography using Diaion HP20 and was then sequentially eluted with water, 30\% $\mathrm{EtOH}$, and $95 \%$ EtOH. The first two fractions $\left(\mathrm{H}_{2} \mathrm{O}\right.$ and $\left.30 \% \mathrm{EtOH}\right)$ were combined, concentrated, and spray-dried to produce the NSF. The last fraction $(95 \% \mathrm{EtOH})$ was concentrated and spray-dried to produce the SF. The extraction procedure for the NFP from KRGE was carried out via EtOH precipitation, enzyme hydrolysis, and size exclusion chromatography as previously described [43]. Briefly, 95\% EtOH was added to the KRGE for precipitation. The solution was treated with $\alpha$-amylase and amyloglucosidase, and the enzyme reaction was then quenched. Following this, 4 volumes of $80 \%$ EtOH were then added to precipitate the polysacchariderich fraction. The polysaccharide-rich fraction was treated with pectinesterase and hydrolyzed with polygalacturonase. The lysates were eventually prepared as the dried NFP after lyophilization.

2.3. Characterization of KRGE Fractions. The ingredients of KRGE powder were determined as follows: ginsenoside and acidic polysaccharide (AP) content [36]. The analyses of ginsenoside [44] and AP [45] were based on the respective method. The analysis of all the contents was performed as previously described [36]. Briefly, to analyze ginsenosides, the samples was extracted with methanol in an ultrasonicator and then subjected to centrifugal separation. The final filtered samples were injected into a Waters ACQUITY UPLC system (Waters, Milford, MA, USA). Eleven ginsenosides were detected using a UPLC Photodiode Array system 
(Waters). To analyze the AP content, we used the carbazolesulfuric acid method. The ginsenoside and AP content of KRGE and its fractions are shown in Table 1.

2.4. Expression and Purification of Recombinant Tau K18. In this study, we used a recombinant K18 fragment which contains four repeats of a critical binding domain associated with the aggregation of tau which thus aggregates faster than the full-length tau $[46,47]$. The tau K18 fragment (129 amino acids), which was cloned from fulllength human tau (hTau40), was expressed in and purified from Escherichia coli BL21 (DE3) cells. Tau K18 was commercially purified by BioProgen (Daejeon, Republic of Korea). The amino acid sequence of tau K18 is as follows: QTAPVPMPDL KNVKSKIGST ENLKHQPGGG KVQIINKKLD LSNVQSKCGS KDNIKHVPGG GSVQIVYKPV DLSKVTSKCG SLGNIHHKPG GGQVEVKSEK LDFKDRVQSK IGSLDNITHV PGGGNKKIE. The DNA sequence of tau K18 was obtained using codon optimization performed by Bioneer (Figure S2). The purity of the proteins was analyzed by $20 \%$ sodium dodecyl sulfatepolyacrylamide gel electrophoresis (Figure S3).

2.5. Evaluation of the Effect of KRGE and Its Fractions on Tau K18 Aggregation. The ThT assay was performed to investigate the effect of KRGE and its fractions on tau aggregation. ThT is known to bind $\beta$-sheet-rich protein aggregates and produce fluorescence [48]. To induce tau aggregation, synthetic tau K18 was dissolved in PBS (pH 7.4). Polymerization of the tau protein was induced by preparing in a $1 \mathrm{~mL}$ solution containing PBS (pH7.4), $1 \mathrm{mg} / \mathrm{mL}$ tau K18, $0.1 \mathrm{mg} / \mathrm{mL}$ heparin, and $100 \mu \mathrm{M}$ DTT. Various concentrations of KRGE $(10,50,100,250$, and $500 \mu \mathrm{g} / \mathrm{mL})$, methylene blue solution (positive control), and KRGE fractions (SF, NSF, and NFP; $10-500 \mu \mathrm{g} / \mathrm{mL}$ ) were incubated with the aggregation mixture for 21 hours (Figure S4). After the incubation period, ThT solution $(15 \mu \mathrm{M}$ in $50 \mathrm{mM}$ glycine buffer, $\mathrm{pH}$ 8.9) was added to a 96-well plate containing samples and the aggregation mixture, and the mixture was incubated for 3 hours. The fluorescence intensity of ThT was measured at excitation and emission wavelengths of 440 and $484 \mathrm{~nm}$ with a SpectraMax iD3 Multi-Mode Microplate Reader (Molecular Devices, San Jose, CA, USA).

The Tecnai G2 spirit TWIN transmission electron microscope (TEM) (Field Electron and Ion Company, Hillsboro, OR, USA) was used for the imaging of tau K18 aggregation. Tau K18 $(1 \mathrm{mg} / \mathrm{mL})$ in PBS (pH 7.4) was incubated with $0.1 \mathrm{mg} / \mathrm{mL}$ heparin and $100 \mu \mathrm{M}$ DTT at $37^{\circ} \mathrm{C}$ for 24 hours with the $500 \mu \mathrm{g} / \mathrm{mL}$ KRGE, SF, NSF, or NFP. The tau K18 sample was placed on a 400-mesh collodion-carbon-coated copper grid (Veco B.V., Eerbeek, Netherlands) and adsorbed for $1 \mathrm{~min}$. Then, the grid was stained with $2.5 \%$ uranyl acetate for $1 \mathrm{~min}$, and excess liquid at the border of the grid was removed by touching it with filter paper. Tau K18 images were captured at $120 \mathrm{kV}$. Length of the tau K18 aggregates was quantified by ImageJ software (NIH, Bethesda, MD, USA).

2.6. Evaluation of the Effect of KRGE and Its Fractions on the Dissociation of Tau K18 Aggregates. The ThT assay was
TABLE 1: Characterization of Korean red ginseng root extract (KRGE) fractions.

\begin{tabular}{lccccc}
\hline & & KRGE & SF & NSF & NFP \\
\hline & Rb1 & 6.23 & 101.79 & ND & ND \\
& Rb2 & 2.45 & 35.58 & ND & ND \\
& Rc & 2.94 & 41.74 & ND & ND \\
& Rd & 1.27 & 14.05 & ND & ND \\
& Re & 0.93 & 23.13 & ND & ND \\
Ginsenoside $(\mathrm{mg} / \mathrm{g})$ & Rf & 1.37 & 20.54 & ND & ND \\
& Rg1 & 0.64 & 17.28 & ND & ND \\
& Rg2s & 1.78 & 20.61 & ND & ND \\
& Rg3r & 1.77 & 21.80 & ND & ND \\
& Rg3s & 3.50 & 44.53 & ND & ND \\
& Rh1 & 1.68 & 15.33 & ND & ND \\
& Total & 24.56 & 356.37 & 0.00 & 0.00 \\
AP (mg/g) & & 98.46 & 5.09 & 98.02 & 438.08 \\
\hline
\end{tabular}

AP: acidic polysaccharide; ND: not detected; NSF: nonsaponin fraction; NFP: nonsaponin fraction with rich polysaccharide; SF: saponin fraction.

performed to investigate the effect of KRGE and its fractions on the dissociation of preformed tau aggregates. To induce tau aggregation, purified $\mathrm{K} 18(1 \mathrm{mg} / \mathrm{mL})$ in phosphatebuffered saline ( $\mathrm{pH} 7.4$ ) was incubated with $0.1 \mathrm{mg} / \mathrm{mL}$ heparin and $100 \mu \mathrm{M}$ DTT at $37^{\circ} \mathrm{C}$ for 24 hours (Figure S4). KRGE and KRGE fractions, including the SF, NSF, and NFP, were added to the aggregation mixture after 21 hours of incubation. At the end of the incubation period, ThT ( $15 \mu \mathrm{M}$ in $50 \mathrm{mM}$ glycine buffer, $\mathrm{pH}$ 8.9) was added to a 96 well plate and incubated for 3 hours. The fluorescence intensity of tau-bound $\mathrm{ThT}$ was measured at $\mathrm{Ex} / \mathrm{Em}=440$ $\mathrm{nm} / 484 \mathrm{~nm}$ with a SpectraMax iD3 Multi-Mode Microplate Reader (Molecular Devices).

2.7. Evaluation of the Effect of KRGE on $A \beta_{1-42}$ Aggregation. The ThT assay was performed to investigate the effect of KRGE on $\mathrm{A} \beta_{1-42}$ aggregation. To induce $\mathrm{A} \beta_{1-42}$ aggregation, synthetic $\mathrm{A} \beta$ was diluted in distilled water to a final concentration of $25 \mu \mathrm{M}$. Various concentrations of KRGE (100, 250, and $500 \mu \mathrm{g} / \mathrm{mL}$ ) and morin (positive control; $100 \mu \mathrm{M}$ ) [4951] were incubated with $A \beta_{1-42}$ peptide solution for 3 hours at $37^{\circ} \mathrm{C}$. After incubation, ThT solution $(15 \mu \mathrm{M}$ in $50 \mathrm{mM}$ glycine buffer, $\mathrm{pH}$ 8.9) was added to a 96-well plate and incubated for 3 hours. The fluorescence intensity of ThT was measured at $\mathrm{Ex} / \mathrm{Em}=440 \mathrm{~nm} / 484 \mathrm{~nm}$ with a SpectraMax iD3 Multi-Mode Microplate Reader (Molecular Devices).

2.8. Statistical Analysis. All statistical analyses were conducted using GraphPad Prism 7.0 software (GraphPad Software, Inc., La Jolla, CA, USA). Data are presented as mean \pm standard error of the mean. The KolmogorovSmirnov test was used to evaluate the normality of the data. When the Kolmogorov-Smirnov test showed no significant differences, Levene's test was performed for analysis of variance between groups. To compare data between three or more groups, one-way analysis of variance followed by Tukey's post hoc test was used. Data which were not 


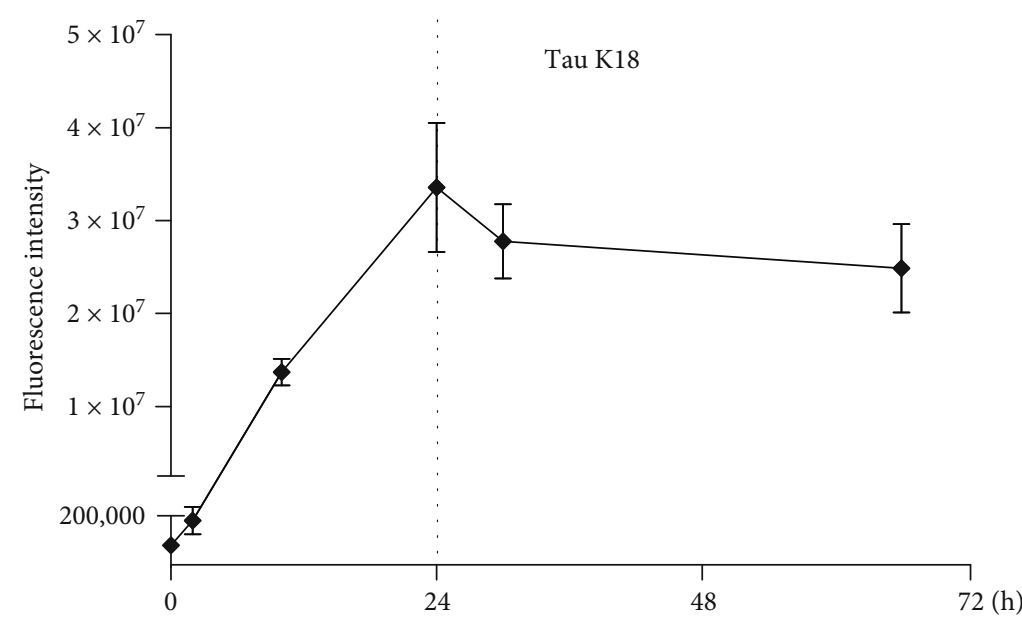

(a)

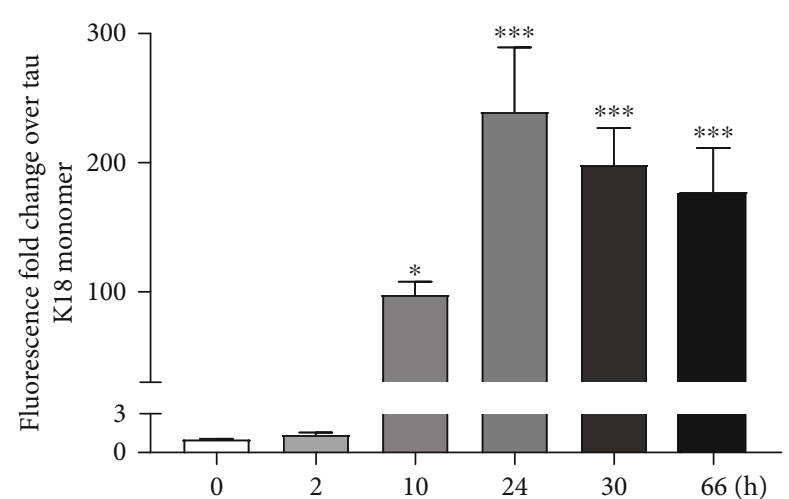

(b)

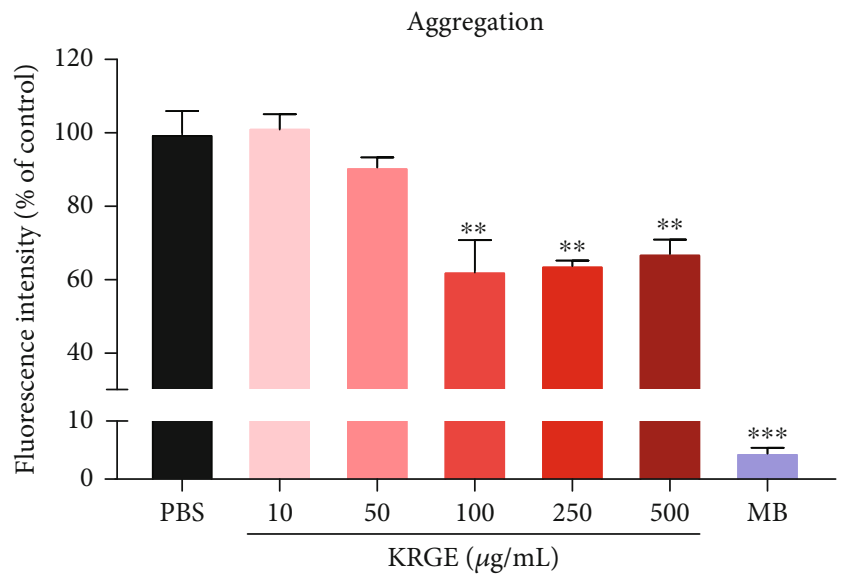

(c)

Dissociation

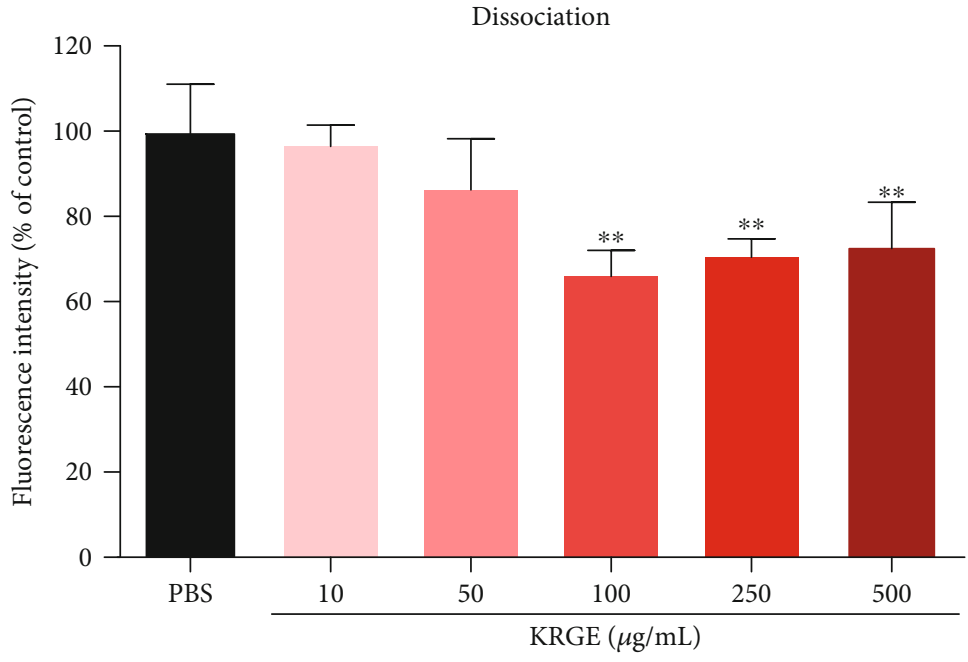

(d)

FIgURE 1: The effect of Korean red ginseng extract (KRGE) on the aggregation and dissociation of tau K18. (a) The aggregation of tau K18 fragments was monitored using the thioflavin T (ThT) assay for 72 hours. (b) The fold changes of the aggregated tau were analyzed by assessing the fluorescence intensity of ThT and normalized to tau K18 monomer. (c) The fluorescence of ThT in the presence and absence of KRGE and methylene blue (MB; $100 \mu \mathrm{M})$. (d) The dissociation of preformed tau aggregates was analyzed by assessing the fluorescence intensity of ThT in the presence and absence of KRGE. Values represent the mean \pm standard error of the mean of three independent experiments. Statistical significance was determined by one-way analysis of variance followed by Tukey's multiple comparison test. ${ }^{*} p<0.05$; ${ }^{* *} p<0.01 ;{ }^{* * *} p<0.001$. 


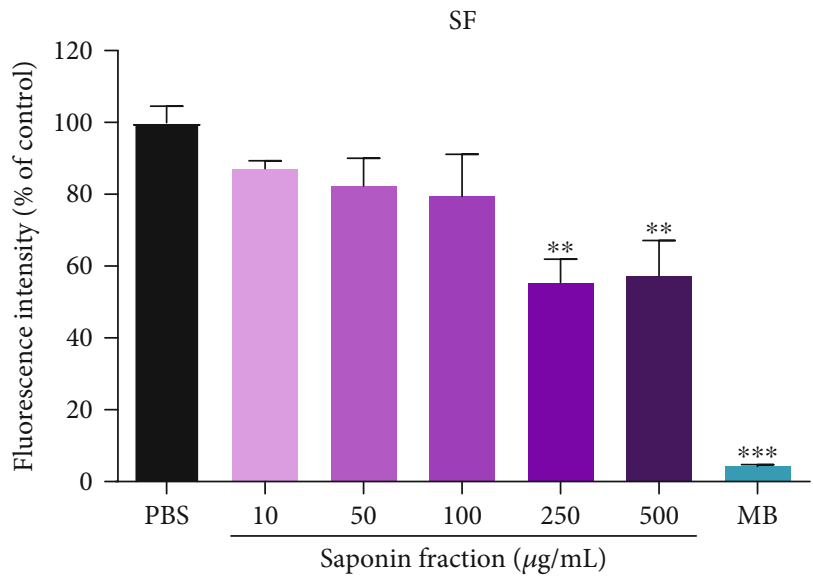

(a)

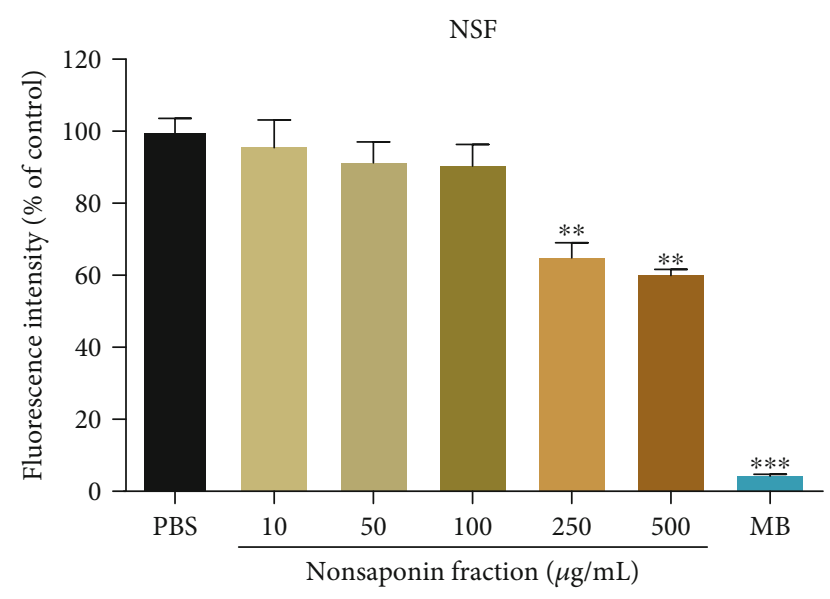

(b)

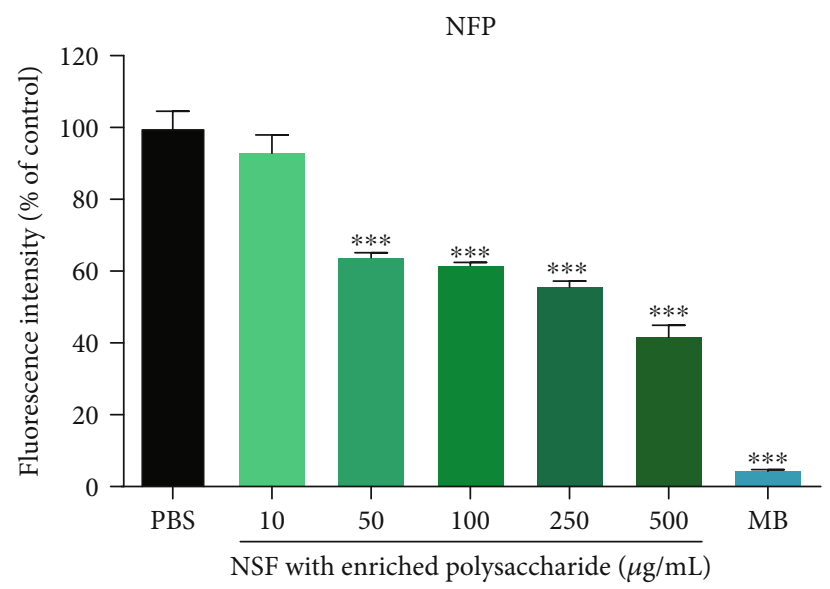

(c)

FIGURE 2: The inhibitory effect of Korean red ginseng extract (KRGE) fractions on tau K18 aggregation. Tau aggregation was analyzed by assessing the fluorescence intensity of thioflavin T (ThT) in the presence and absence of (a) the saponin fraction (SF), (b) the nonsaponin fraction (NSF), and (c) the NSF with rich polysaccharide (NFP). Methylene blue (MB; $100 \mu \mathrm{M})$, a known inhibitor of tau aggregation, was used as a positive control. Values represent the mean \pm standard error of the mean of three independent experiments. Statistical significance was determined by one-way analysis of variance followed by Tukey's multiple comparison test. ${ }^{* *} p<0.01 ;{ }^{* * *} p<0.001$.

normally distributed were analyzed by the Kruskal-Wallis test followed by Dunn's post hoc test. A $p$ value $<0.05$ indicated statistical significance. $\mathrm{IC}_{50}$ and $\mathrm{DC}_{50}$ values were determined by nonlinear regression analysis.

\section{Results}

3.1. Characterization of KRGE and KRGE Fractions. According to the previous results of the component analysis of KRGE powder, total ginsenoside content was $24.56 \mathrm{mg} / \mathrm{g}$ and AP content was $98.46 \mathrm{mg} / \mathrm{g}$ [36]. In the present study, the total ginsenoside content of the SF was almost 14.5 times $(356.37 \mathrm{mg} / \mathrm{g})$ that of the KRGE powder $(24.56 \mathrm{mg} / \mathrm{g})$ (Table 1). No ginsenosides were detected in the NSF and NFP. The NFP showed an 86-fold higher AP content than the SF $(438.08 \mathrm{mg} / \mathrm{g}$ and $5.09 \mathrm{mg} / \mathrm{g}$, respectively). KRGE powder $(98.46 \mathrm{mg} / \mathrm{g})$ and the NSF $(98.02 \mathrm{mg} / \mathrm{g})$ had similar AP content. The composition of all samples is summarized in Table 1 .
3.2. KRGE Inhibits Tau Aggregation and Induces Tau Dissociation. We investigated the effect of KRGE on tau aggregation and disassociation using the ThT assay (Figure S4). Prior to treatment with KRGE, tau K18 fragments were incubated with heparin for 72 hours to monitor alterations in ThT fluorescence intensity according to the aggregation of tau K18 (Figure 1(a)). In addition, the fold change was analyzed to confirm the aggregated tau for the tau monomer over time (Figure 1(b)). After incubating tau K18 for 24 hours, the highest intensity was observed by 239.4-folds. Next, tau K18 fragments were incubated with heparin in the presence or absence of KRGE. We observed that $\operatorname{KRGE}(10,50,100,250$, and $500 \mu \mathrm{g} / \mathrm{mL})$ significantly inhibited tau aggregation (Figure 1(c)) Since methylene blue is known to hinder the aggregation of tau [52], we used it as a positive control. In addition, the intensity of ThT fluorescence was significantly reduced when KRGE $(10,50,100,250$, and $500 \mu \mathrm{g} / \mathrm{mL})$ was incubated with preformed aggregates of tau K18 (Figure 1(d)). However, KRGE treatment did not affect the aggregation of 


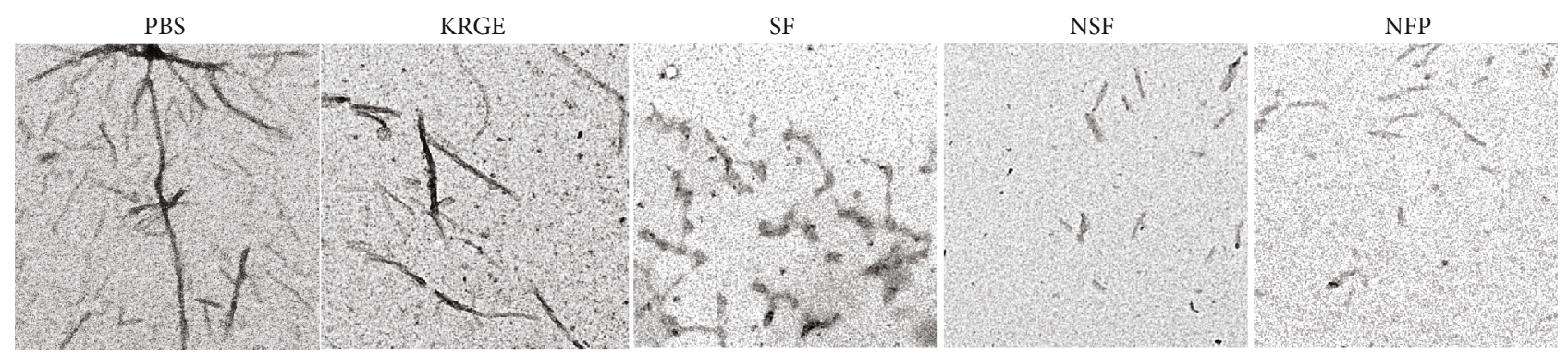

(a)

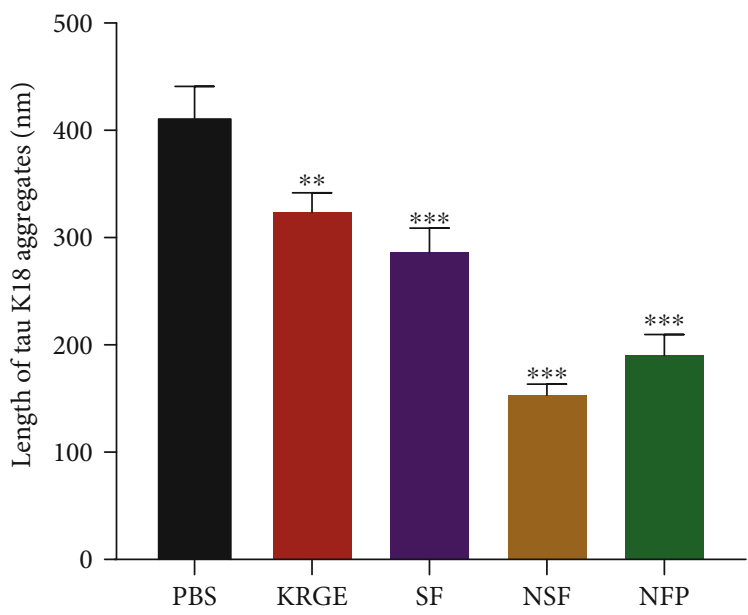

(b)

Figure 3: The effect of KRGE and KRGE fractions on the aggregation of tau K18 using the transmission electron microscopy (TEM). (a) Images of tau K18 aggregates in the absence or presence of $500 \mu \mathrm{g} / \mathrm{mL}$ KRGE or KRGE fractions (scale bar $=200 \mathrm{~nm}$ ). (b) The length of the tau K18 aggregates was quantified in TEM images. Values represent the mean \pm standard error of the mean. Statistical significance was determined by one-way analysis of variance followed by Tukey's multiple comparison test. ${ }^{* *} p<0.01 ;{ }^{* * *} p<0.001$.

monomeric $\mathrm{A} \beta_{1-42}$ (Figure S5). These results indicate that KRGE can modulate tau aggregation and disassociation.

\subsection{Fractions of KRGE Interfere with Tau K18 Aggregation.} Next, we examined which fractions of KRGE have a direct effect on tau aggregation using the ThT assay. The SF of KRGE $(250$ and $500 \mu \mathrm{g} / \mathrm{mL})$ significantly inhibited the aggregation of tau K18 (Figure 2(a)). In addition, incubation with the NSF (250 and $500 \mu \mathrm{g} / \mathrm{mL}$ ) also significantly inhibited the aggregation of tau (Figure 2(b)). Moreover, treatment with the NFP inhibited tau aggregation in a concentrationdependent manner (Figure 2(c)). Methylene blue was used as a positive control and significantly reduced tau aggregation. To confirm the inhibitory effect of KRGE fractions on tau K18 aggregation, tau K18 aggregates were imaged with a TEM and the length of the aggregates was quantified (Figure 3(a)). Significant reduction in the length of tau K18 aggregates was observed after treatment with KRGE or KRGE fractions at a dose of $500 \mu \mathrm{g} / \mathrm{mL}$ (Figure 3(b)). There was no significant difference in length of tau K18 aggregates between NSF and NFP. Taken together, we demonstrated that KRGE fractions can effectively prevent tau aggregation.

3.4. KRGE Fractions Dissociate Preformed Tau K18 Aggregates. Furthermore, we examined whether KRGE frac- tions can promote the disassociation of tau aggregates using the ThT assay. Tau K18 fragments were incubated with heparin for 24 hours, and preformed tau aggregates were then incubated with KRGE fractions (SF, NSF, and NFP) for 21 hours (Figure S4). ThT fluorescence was significantly reduced by incubation of the SF with tau K18 fragments (Figure 4(a)). Additionally, we found that incubation of tau K18 fragments with the NSF caused a significant decrease in the fluorescence intensity of ThT (Figure 4(b)). The ThT assay revealed that the NFP also stimulated tau dissociation, as demonstrated by the observed decrease in ThT fluorescence (Figure 4(c)). Overall, our data showed that KRGE fractions are able to disassociate preformed tau fibrils.

\section{Discussion}

Ginseng is known to have beneficial effects in neurodegenerative diseases involving the pathological aggregation of tau in the brain [53]. Among the various types of processed ginseng, $R G$ has been reported to improve cognitive function in both rodents and humans [54-56]. In particular, the antidementia effect of RG has been demonstrated in animal models of $\mathrm{AD}$ and $\mathrm{AD}$ patients [36, 37, 39, 40]. Therefore, the effects of ginsenosides, the main active components of 


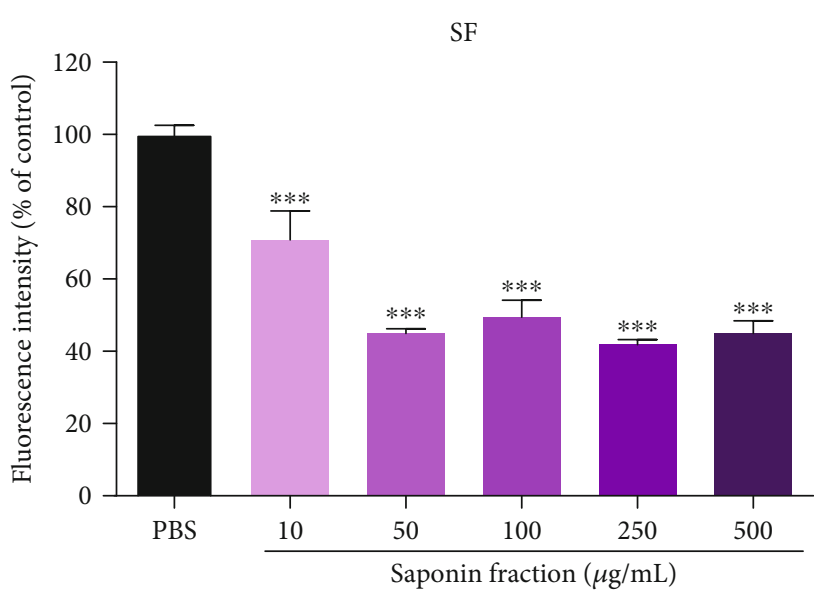

(a)

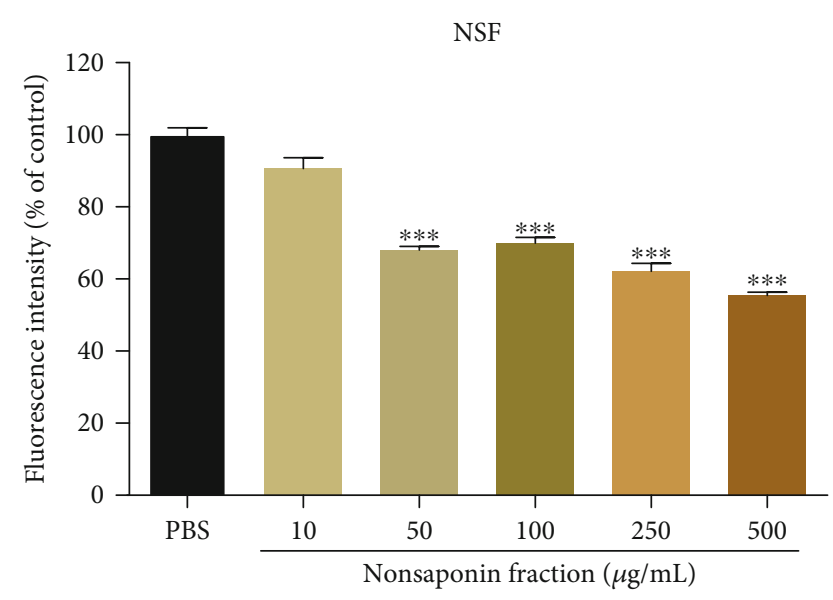

(b)

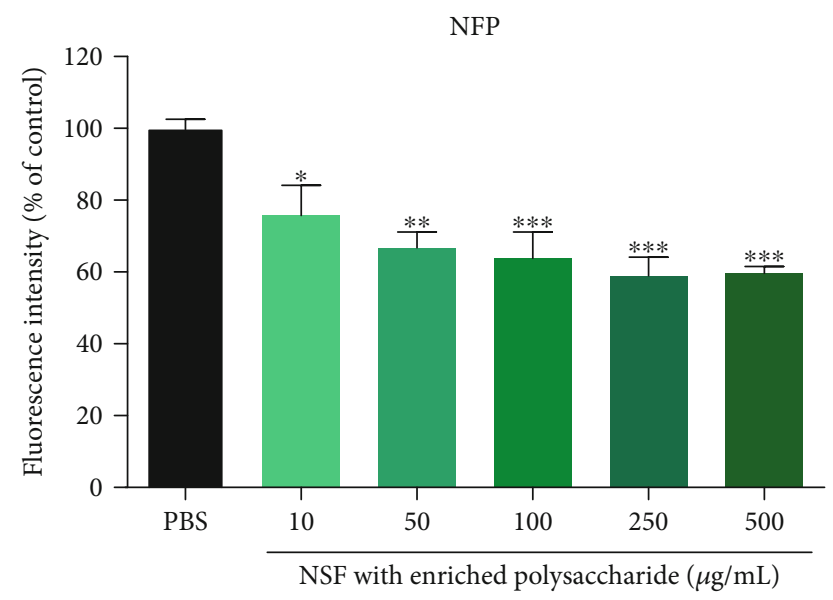

(c)

FIGURE 4: The stimulatory effect of Korean red ginseng extract (KRGE) fractions on tau K18 dissociation. Tau dissociation was analyzed by assessing the fluorescence intensity of thioflavin T (ThT) in the presence and absence of (a) the saponin fraction (SF), (b) the nonsaponin fraction (NSF), and (c) the NSF with rich polysaccharide (NFP). Values represent the mean \pm standard error of the mean of three independent experiments. Statistical significance was determined by one-way analysis of variance followed by Tukey's multiple comparison test. ${ }^{*} p<0.05 ;{ }^{* *} p<0.01 ;{ }^{* * *} p<0.001$.

RG, on AD-related pathology such as neuroinflammation $[57,58]$, mitochondrial dysfunction [59-62], and amyloidogenic mechanism [63-66] have previously been studied. However, to the best of our knowledge, only one study has examined the effect of ginsenosides on tau pathology [42]. Moreover, although polysaccharides and nonsaponin compounds are the main constituents of RG, their effect on tau pathology has not yet been studied. To investigate whether RG can alleviate tau pathology, we evaluated the effect of KRGE and its components on tau aggregation and dissociation using tau K18 fragments and ThT fluorescence. Tau K18 fragments comprise four tubulin-binding repeats and make up the paired helical filament core in the microtubule-binding domain of full-length $\mathrm{hTau}_{441}(4 \mathrm{R} 2 \mathrm{~N})$ (Figure 5). These fragments have been used in numerous studies to characterize tau aggregation and dissociation in vitro [46, 67-71].

Remarkably, our results demonstrated that treatment with KRGE can modulate the aggregation and dissociation of tau K18 fragments (Figures 1-4). Furthermore, to determine which components of KRGE contribute to its antiaggregation effect, we evaluated the effect of KRGE fractions, including the SF, NSF, and NFP, on the aggregation of tau K18 fragments. All KRGE fractions inhibited the aggregation of tau K18 fragments at high concentrations $(250$ and $500 \mu \mathrm{g} / \mathrm{mL}$ ). Among the KRGE fractions, the NFP most potently inhibited tau aggregation (Figure 2). Given the proportion of polysaccharides in each of the KRGE fractions (Table 1), the antiaggregation effect of KRGE on tau appears to primarily derive from the NFP. Moreover, we investigated the effect of KRGE fractions on the disassociation of tau K18 aggregates. The SF most effectively induced the dissociation of tau K18 fragments, and the NFP also promoted tau dissociation even at the lowest concentration studied $(10 \mu \mathrm{g} / \mathrm{mL})$ (Figure 4). Based on these results, we derived the $\mathrm{IC}_{50}$ and $\mathrm{DC}_{50}$ of $\mathrm{KRGE}$ extract on the aggregation and dissociation of tau K18 (Table 2). These results suggest that KRGE fractions may modulate the aggregation and dissociation of tau via different mechanisms. 


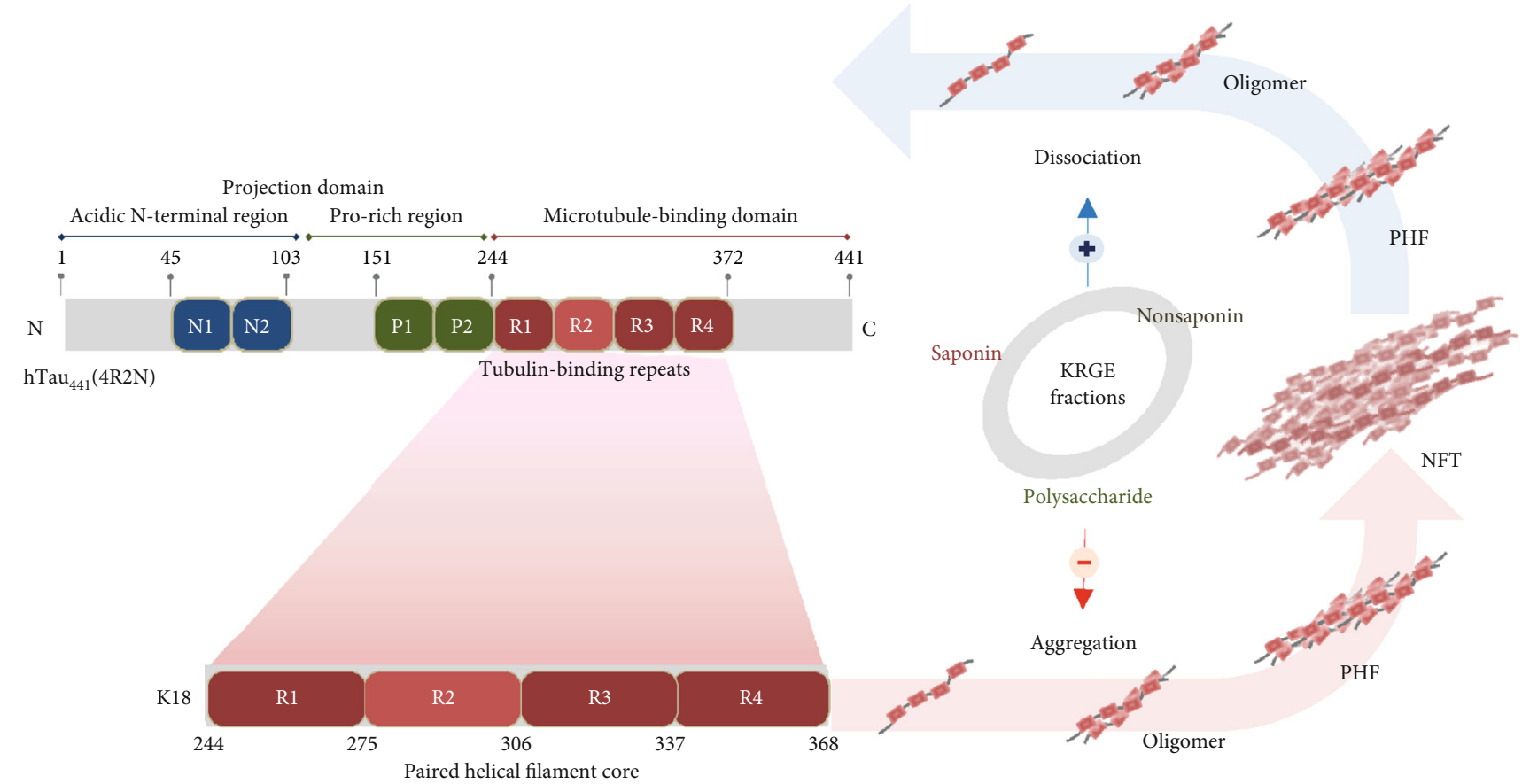

Figure 5: A schematic illustration of the structure of tau K18 and the modulatory effects of KRGE fractions on tau K18 aggregation/dissociation. KRGE and its constituents exhibited modulatory effects on tau aggregation and dissociation. KRGE: Korean red ginseng extract; NFT: neurofibrillary tangle; PHF: paired helical filament.

TABle 2: Determination of $\mathrm{IC}_{50}$ and $\mathrm{DC}_{50}$ values of the Korean red ginseng root extract (KRGE) for aggregation and dissociation of tau K18.

\begin{tabular}{lcc}
\hline & Aggregation & Dissociation \\
\hline & $\mathrm{IC}_{50}(\mu \mathrm{g} / \mathrm{mL})$ & $\mathrm{DC}_{50}(\mu \mathrm{g} / \mathrm{mL})$ \\
KRGE & $545.0 \pm 124.4$ & $713.5 \pm 158.3$ \\
$\mathrm{SF}$ & $395.1 \pm 89.02$ & $100.2 \pm 28.87$ \\
$\mathrm{NSF}$ & $645.4 \pm 137.7$ & $370.3 \pm 58.65$ \\
$\mathrm{NFP}$ & $179.3 \pm 25.51$ & $271.8 \pm 69.44$ \\
\hline
\end{tabular}

NSF: nonsaponin fraction; NFP: nonsaponin fraction with rich polysaccharide; SF: saponin fraction.

The toxicity of tau aggregates is controversial and is thought to depend on the form of aggregate and the microenvironment [72]. In a previous study, mice with human tau mutations exhibited behavioral deficits, neuronal loss, and progressive NFT deposition with age. Reducing the expression of mutant tau ameliorated tau-related pathological symptoms such as neuronal loss and memory, but NFTs continued to accumulate in the brain [73]. These findings suggest that neurodegeneration and cognitive dysfunction associated with tauopathy are not NFT-dependent. Rather, soluble tau aggregates such as oligomers may directly contribute to the progression of tau-related pathology. However, as mentioned above, many studies have demonstrated that inhibiting tau aggregation or promoting tau dissociation may also contribute to alleviating tauopathy. Despite this controversy, targeting tau aggregates is a therapeutic strategy that accounts for a large proportion of clinical trials for the treatment of $\mathrm{AD}$ [29]. In addition, a number of studies demonstrated that inhibiting tau aggregation or promoting the dissociation of tau aggregates can have a beneficial effect through various mechanisms such as propagation, degradation, and sequestration (Figure 6). Self-aggregating hyperphosphorylated tau has been reported to sequester normal tau during the aggregation process [74-76]. Therefore, inhibiting tau aggregation may reduce normal tau sequestration by hyperphosphorylated tau and contribute to microtubule stabilization. Furthermore, because abnormal tau aggregates are known to undergo autophagic and proteasomal degradation [77-79], dissociating insoluble forms of tau aggregates, such as NFTs or paired helical filaments, into smaller soluble forms may contribute to the removal of abnormally phosphorylated tau. Moreover, inhibiting tau aggregation and promoting the dissociation of tau aggregates may suppress intercellular tau propagation, which is known to induce neuroinflammation by activating astrocytes and microglia $[80,81]$. These studies suggest that inhibiting tau aggregation or promoting tau dissociation may be promising strategies for the treatment of tauopathies (Figure 6).

In our previous study, administration of KRGE reduced $\mathrm{A} \beta$ accumulation in the brains of $\mathrm{A} \beta$-overexpressing transgenic mice [36]. Thus, we analyzed the effect of KRGE on $\mathrm{A} \beta$ aggregation through $\mathrm{ThT}$ fluorescence to determine whether KRGE alleviated $\mathrm{A} \beta$ accumulation by directly interacting with the $\mathrm{A} \beta$ peptide (Figure S5). KRGE did not directly affect the aggregation of $\mathrm{A} \beta_{1-42}$, indicating that the reduction of $\mathrm{A} \beta$ accumulation shown in the brains of $\mathrm{A} \beta$ overexpressing transgenic mice may not be mediated through direct inhibition of $\mathrm{A} \beta$ accumulation, but through alternative pathways, including inhibition of mitochondrial 


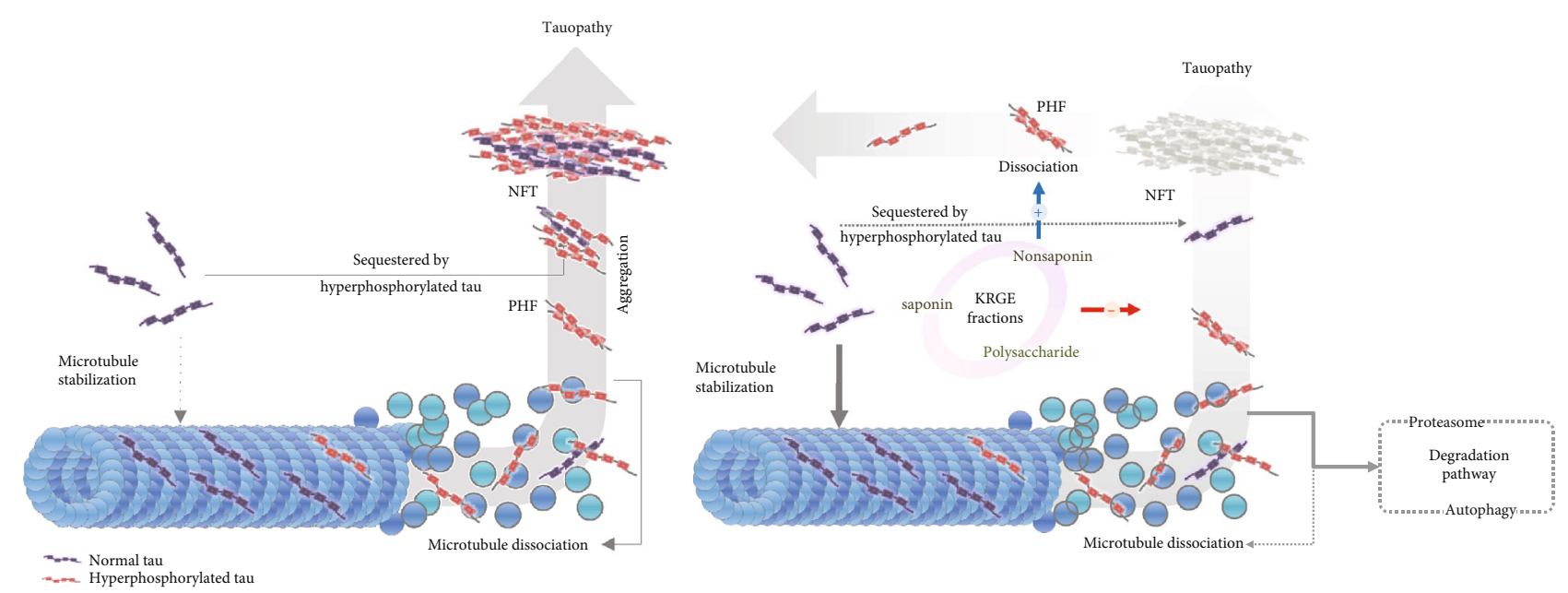

FIgURE 6: Tau pathomechanisms and proposed mechanism underlying the modulatory effect of KRGE and its fractions on tau K18 aggregation/dissociation. KRGE: Korean red ginseng extract; NFT: neurofibrillary tangle; PHF: paired helical filament.

dysfunction, modulation of the $\mathrm{A} \beta$ production pathway, or activation of $\mathrm{A} \beta$ degradation/clearance.

In future work, we plan to evaluate the in vivo efficacy of KRGE and its constituents in animal models that exhibit tau-related pathology such as the triple-transgenic mouse model of $\mathrm{AD}$, which exhibits both $\mathrm{A} \beta$ and tau pathology, and P301S tau transgenic mice (a human tauopathy model). Furthermore, we intend to conduct a future study in order to further characterize KRGE fractions and identify the principal components which modulate the aggregation and dissociation of tau.

\section{Conclusions}

Our findings demonstrate that KRGE and its constituents can regulate the aggregation and dissociation of tau and thus may be beneficial for the treatment of neurodegenerative diseases associated with tauopathy.

\section{Data Availability}

The data used to support the findings of this study are available from the corresponding author upon request.

\section{Conflicts of Interest}

The authors declare that there is no conflict of interest regarding the publication of this paper.

\section{Authors' Contributions}

Soo Jung Shin, Yong Ho Park, and Seong Gak Jeon contributed equally to this work.

\section{Acknowledgments}

This work was supported by the Korea Ginseng Corporation (KGC).

\section{Supplementary Materials}

Supplementary Figure 1: composition of Korean red ginseng extract: saponin fraction, nonsaponin fraction, and nonsaponin fraction (NSF) with rich polysaccharide. Supplementary Figure 2: amino acid and DNA sequences for synthesis of recombinant tau K18 fragments. Supplementary Figure 3: purification of recombinant tau K18. The recombinant tau K18 was confirmed by sodium dodecyl sulfatepolyacrylamide gel electrophoresis. Marker indicates the molecular weight of the protein, and the rest of the lanes contain purified protein. Supplementary Figuer 4: overview of the thioflavin T (ThT) assay for evaluating the effect of Korean red ginseng extract (KRGE) and KRGE fractions (KRGFs) on the aggregation and dissociation of tau K18 fragments. Supplementary Figure 5: evaluation of the effect of Korean red ginseng extract (KRGE) on $\mathrm{A} \beta_{1-42}$ aggregation. (a) Fluorescence intensity during the thioflavin $\mathrm{T}$ (ThT) assay. Monomeric $A \beta_{1-42}$ was incubated for 5 hours to allow for oligomerization. (b) The ThT assay was performed to examine the effect of KRGE on $\mathrm{A} \beta_{1-42}$ aggregation. When $\mathrm{A} \beta_{1-42}$ was incubated in the presence of morin, a well-known inhibitor of $\mathrm{A} \beta$ aggregation, $\mathrm{A} \beta_{1-42}$ aggregation was dramatically reduced. However, no significant change in $\mathrm{A} \beta_{1-42}$ aggregation was observed after incubation with KRGE. Values represent the mean \pm standard error of the mean of three independent experiments. Statistical significance was determined by one-way analysis of variance followed by Tukey's multiple comparison test. ${ }^{* * *} p<0.001$. (Supplementary Materials)

\section{References}

[1] M. D. Weingarten, A. H. Lockwood, S. Y. Hwo, and M. W. Kirschner, "A protein factor essential for microtubule assembly," Proceedings of the National Academy of Sciences of the United States of America, vol. 72, no. 5, pp. 18581862, 1975.

[2] D. W. Cleveland, S. Y. Hwo, and M. W. Kirschner, "Purification of tau, a microtubule-associated protein that induces 
assembly of microtubules from purified tubulin," Journal of Molecular Biology, vol. 116, no. 2, pp. 207-225, 1977.

[3] A. Himmler, D. Drechsel, M. W. Kirschner, and D. W. Martin Jr., "Tau consists of a set of proteins with repeated C-terminal microtubule-binding domains and variable $\mathrm{N}$-terminal domains," Molecular and Cellular Biology, vol. 9, no. 4, pp. 1381-1388, 1989.

[4] M. Goedert, M. G. Spillantini, R. Jakes, D. Rutherford, and R. A. Crowther, "Multiple isoforms of human microtubuleassociated protein tau: sequences and localization in neurofibrillary tangles of Alzheimer's disease," Neuron, vol. 3, no. 4, pp. 519-526, 1989.

[5] A. D. C. Alonso, A. Mederlyova, M. Novak, I. Grundke-Iqbal, and K. Iqbal, "Promotion of hyperphosphorylation by frontotemporal dementia tau mutations," The Journal of Biological Chemistry, vol. 279, no. 33, pp. 34873-34881, 2004.

[6] A. D. Alonso, T. Zaidi, M. Novak, H. S. Barra, I. GrundkeIqbal, and K. Iqbal, "Interaction of tau isoforms with Alzheimer's disease abnormally hyperphosphorylated tau and in vitro phosphorylation into the disease-like protein," The Journal of Biological Chemistry, vol. 276, no. 41, pp. 3796737973, 2001.

[7] T. Arendt and T. Bullmann, "Neuronal plasticity in hibernation and the proposed role of the microtubule-associated protein tau as a "master switch" regulating synaptic gain in neuronal networks," American Journal of Physiology. Regulatory, Integrative and Comparative Physiology, vol. 305, no. 5, pp. R478-R489, 2013.

[8] J. P. Brion, J. N. Octave, and A. M. Couck, "Distribution of the phosphorylated microtubule-associated protein tau in developing cortical neurons," Neuroscience, vol. 63, no. 3, pp. 895-909, 1994.

[9] A. D. Alonso, L. S. Cohen, C. Corbo et al., "Hyperphosphorylation of tau associates with changes in its function beyond microtubule stability," Frontiers in Cellular Neuroscience, vol. 12, p. 338, 2018.

[10] N. S. Honson and J. Kuret, "Tau aggregation and toxicity in tauopathic neurodegenerative diseases," Journal of Alzheimer's Disease, vol. 14, no. 4, pp. 417-422, 2008.

[11] V. Zhukareva, D. Mann, S. Pickering-Brown et al., "Sporadic Pick's disease: a tauopathy characterized by a spectrum of pathological ? isoforms in gray and white matter," Annals of Neurology, vol. 51, no. 6, pp. 730-739, 2002.

[12] X. Zhang, F. Gao, D. Wang et al., "Tau pathology in Parkinson's disease," Frontiers in Neurology, vol. 9, p. 809, 2018.

[13] K. Iqbal, F. Liu, C. X. Gong, and I. Grundke-Iqbal, "Tau in Alzheimer disease and related tauopathies," Current Alzheimer Research, vol. 7, no. 8, pp. 656-664, 2010.

[14] M. Hutton, C. L. Lendon, P. Rizzu et al., "Association of missense and $5^{\prime}$-splice-site mutations in tau with the inherited dementia FTDP-17," Nature, vol. 393, no. 6686, pp. 702-705, 1998.

[15] G. U. Höglinger, PSP Genetics Study Group, N. M. Melhem et al., "Identification of common variants influencing risk of the tauopathy progressive supranuclear palsy," Nature Genetics, vol. 43, no. 7, pp. 699-705, 2011.

[16] C. M. Cowan and A. Mudher, "Are tau aggregates toxic or protective in tauopathies?," Frontiers in Neurology, vol. 4, 2013.

[17] I. Khlistunova, J. Biernat, Y. Wang et al., "Inducible expression of tau repeat domain in cell models of tauopathy,"
The Journal of Biological Chemistry, vol. 281, no. 2, pp. 1205-1214, 2006.

[18] K. R. Patterson, S. M. Ward, B. Combs et al., "Heat shock protein 70 prevents both tau aggregation and the inhibitory effects of preexisting tau aggregates on fast axonal transport," Biochemistry, vol. 50, no. 47, pp. 10300-10310, 2011.

[19] C. A. Lasagna-Reeves, D. L. Castillo-Carranza, U. Sengupta, A. L. Clos, G. R. Jackson, and R. Kayed, "Tau oligomers impair memory and induce synaptic and mitochondrial dysfunction in wild-type mice," Molecular Neurodegeneration, vol. 6, no. 1, p. 39, 2011.

[20] C. A. Lasagna-Reeves, D. L. Castillo-Carranza, M. J. GuerreroMuñoz, G. R. Jackson, and R. Kayed, "Preparation and characterization of neurotoxic tau oligomers," Biochemistry, vol. 49, no. 47, pp. 10039-10041, 2010.

[21] M. Iba, J. L. Guo, J. D. McBride, B. Zhang, J. Q. Trojanowski, and V. M. Y. Lee, "Synthetic tau fibrils mediate transmission of neurofibrillary tangles in a transgenic mouse model of Alzheimer's-like tauopathy," The Journal of Neuroscience, vol. 33, no. 3, pp. 1024-1037, 2013.

[22] A. de Calignon, M. Polydoro, M. Suarez-Calvet et al., "Propagation of tau pathology in a model of early Alzheimer's disease," Neuron, vol. 73, no. 4, pp. 685-697, 2012.

[23] F. Clavaguera, T. Bolmont, R. A. Crowther et al., "Transmission and spreading of tauopathy in transgenic mouse brain," Nature Cell Biology, vol. 11, no. 7, pp. 909-913, 2009.

[24] B. Frost, R. L. Jacks, and M. I. Diamond, "Propagation of tau misfolding from the outside to the inside of a cell," The Journal of Biological Chemistry, vol. 284, no. 19, pp. 12845-12852, 2009.

[25] H. C. Tai, A. Serrano-Pozo, T. Hashimoto, M. P. Frosch, T. L. Spires-Jones, and B. T. Hyman, "The synaptic accumulation of hyperphosphorylated tau oligomers in Alzheimer disease is associated with dysfunction of the ubiquitin-proteasome system," The American Journal of Pathology, vol. 181, no. 4, pp. 1426-1435, 2012.

[26] K. Eckermann, M. M. Mocanu, I. Khlistunova et al., "The betapropensity of tau determines aggregation and synaptic loss in inducible mouse models of tauopathy," The Journal of Biological Chemistry, vol. 282, no. 43, pp. 31755-31765, 2007.

[27] K. Yanamandra, N. Kfoury, H. Jiang et al., “Anti-tau antibodies that block tau aggregate seeding in vitro markedly decrease pathology and improve cognition in vivo," Neuron, vol. 80, no. 2, pp. 402-414, 2013.

[28] C. Fatouros, G. J. Pir, J. Biernat et al., "Inhibition of tau aggregation in a novel Caenorhabditis elegans model of tauopathy mitigates proteotoxicity," Human Molecular Genetics, vol. 21, no. 16, pp. 3587-3603, 2012.

[29] J. Cummings, K. Blennow, K. Johnson et al., “Anti-tau trials for Alzheimer's disease: a report from the EU/US/CTAD task force," The Journal of Prevention of Alzheimer's Disease, vol. 6, no. 3, pp. 157-163, 2019.

[30] G. Simic, M. Babic Leko, S. Wray et al., "Tau protein hyperphosphorylation and aggregation in Alzheimer's disease and other tauopathies, and possible neuroprotective strategies," Biomolecules, vol. 6, no. 1, p. 6, 2016.

[31] K. T. Choi, "Botanical characteristics, pharmacological effects and medicinal components of Korean Panax ginseng C A Meyer," Acta Pharmacologica Sinica, vol. 29, no. 9, pp. 1109$1118,2008$. 
[32] S. M. Lee, B. S. Bae, H. W. Park et al., "Characterization of Korean red ginseng (Panax ginseng Meyer): history, preparation method, and chemical composition," Journal of Ginseng Research, vol. 39, no. 4, pp. 384-391, 2015.

[33] H. D. Yuan, J. T. Kim, S. H. Kim, and S. H. Chung, "Ginseng and diabetes: the evidences from in vitro, animal and human studies," Journal of Ginseng Research, vol. 36, no. 1, pp. 2739,2012

[34] A. S. T. Wong, C.-M. Che, and K.-W. Leung, "Recent advances in ginseng as cancer therapeutics: a functional and mechanistic overview," Natural Product Reports, vol. 32, no. 2, pp. 256272, 2015.

[35] J. H. Kim, "Cardiovascular diseases and Panax ginseng: a review on molecular mechanisms and medical applications," Journal of Ginseng Research, vol. 36, no. 1, pp. 16-26, 2012.

[36] S. J. Shin, S. G. Jeon, J. I. Kim et al., "Red ginseng attenuates $\mathrm{A} \beta$-induced mitochondrial dysfunction and $\mathrm{A} \beta$-mediated pathology in an animal model of Alzheimer's disease," International Journal of Molecular Sciences, vol. 20, no. 12, p. 3030, 2019.

[37] J. H. Heo, M. H. Park, and J. H. Lee, "Effect of Korean red ginseng on cognitive function and quantitative EEG in patients with Alzheimer's disease: a preliminary study," Journal of Alternative and Complementary Medicine, vol. 22, no. 4, pp. 280-285, 2016.

[38] J. H. Choi, M. Jang, S. Y. Nah, S. Oh, and I. H. Cho, "Multitarget effects of Korean red ginseng in animal model of Parkinson's disease: antiapoptosis, antioxidant, antiinflammation, and maintenance of blood-brain barrier integrity," Journal of Ginseng Research, vol. 42, no. 3, pp. 379-388, 2018.

[39] J. H. Heo, S. T. Lee, K. Chu et al., "An open-label trial of Korean red ginseng as an adjuvant treatment for cognitive impairment in patients with Alzheimer's disease," European Journal of Neurology, vol. 15, no. 8, pp. 865-868, 2008.

[40] J. H. Heo, S. T. Lee, M. J. Oh et al., "Improvement of cognitive deficit in Alzheimer's disease patients by long term treatment with Korean red ginseng," Journal of Ginseng Research, vol. 35, no. 4, pp. 457-461, 2011.

[41] H. J. Kim, S. W. Jung, S. Y. Kim et al., "Panax ginseng as an adjuvant treatment for Alzheimer's disease," Journal of Ginseng Research, vol. 42, no. 4, pp. 401-411, 2018.

[42] Y. Zhang, Z. Pi, F. Song, and Z. Liu, "Ginsenosides attenuate d-galactose- and AlCl3-inducedspatial memory impairment by restoring the dysfunction of the neurotransmitter systems in the rat model of Alzheimer's disease," Journal of Ethnopharmacology, vol. 194, pp. 188-195, 2016.

[43] S. J. Lee, G. In, S.-T. Han, M.-H. Lee, J.-W. Lee, and K.-S. Shin, "Structural characteristics of a red ginseng acidic polysaccharide rhamnogalacturonan I with immunostimulating activity from red ginseng," Journal of Ginseng Research, 2019.

[44] H. W. Park, G. In, S. T. Han et al., "Simultaneous determination of 30 ginsenosides in Panax ginseng preparations using ultra performance liquid chromatography," Journal of Ginseng Research, vol. 37, no. 4, pp. 457-467, 2013.

[45] G. In, N. G. Ahn, B. S. Bae et al., "In situ analysis of chemical components induced by steaming between fresh ginseng, steamed ginseng, and red ginseng," Journal of Ginseng Research, vol. 41, no. 3, pp. 361-369, 2017.

[46] S. Lim, M. M. Haque, D. Kim, D. J. Kim, and Y. K. Kim, "Cellbased models to investigate tau aggregation," Computational and Structural Biotechnology Journal, vol. 12, no. 20-21, pp. 7-13, 2014.

[47] M. M. Haque, D. Kim, Y. H. Yu et al., "Inhibition of tau aggregation by a rosamine derivative that blocks tau intermolecular disulfide cross-linking," Amyloid, vol. 21, no. 3, pp. 185-190, 2014.

[48] L. S. Wolfe, M. F. Calabrese, A. Nath, D. V. Blaho, A. D. Miranker, and Y. Xiong, "Protein-induced photophysical changes to the amyloid indicator dye thioflavin T," Proceedings of the National Academy of Sciences of the United States of America, vol. 107, no. 39, pp. 16863-16868, 2010.

[49] H. Noor, P. Cao, and D. P. Raleigh, "Morin hydrate inhibits amyloid formation by islet amyloid polypeptide and disaggregates amyloid fibers," Protein Science, vol. 21, no. 3, pp. 373$382,2012$.

[50] S. J. Shin, Y. Jeong, S. G. Jeon et al., "Uncaria rhynchophylla ameliorates amyloid beta deposition and amyloid betamediated pathology in 5XFAD mice," Neurochemistry International, vol. 121, pp. 114-124, 2018.

[51] S. Shin, Y.-o. Jeong, S. Jeon et al., "Jowiseungchungtang inhibits amyloid- $\beta$ aggregation and amyloid- $\beta$-mediated pathology in 5XFAD mice," International Journal of Molecular Sciences, vol. 19, no. 12, p. 4026, 2018.

[52] B. Bulic, M. Pickhardt, B. Schmidt, E. M. Mandelkow, H. Waldmann, and E. Mandelkow, "Development of tau aggregation inhibitors for Alzheimer's disease," Angewandte Chemie International Edition, vol. 48, no. 10, pp. 1740-1752, 2009.

[53] K. H. Kim, D. Lee, H. L. Lee, C. E. Kim, K. Jung, and K. S. Kang, "Beneficial effects of Panax ginseng for the treatment and prevention of neurodegenerative diseases: past findings and future directions," Journal of Ginseng Research, vol. 42, no. 3, pp. 239-247, 2018.

[54] H. B. Yeo, H. K. Yoon, H. J. Lee, S. G. Kang, K. Y. Jung, and L. Kim, "Effects of Korean red ginseng on cognitive and motor function: a double-blind, randomized, placebo-controlled trial," Journal of Ginseng Research, vol. 36, no. 2, pp. 190197, 2012.

[55] Y. Lee and S. Oh, "Administration of red ginseng ameliorates memory decline in aged mice," Journal of Ginseng Research, vol. 39, no. 3, pp. 250-256, 2015.

[56] Y. M. Zhong, H. Nishijo, T. Uwano, R. Tamura, K. Kawanishi, and T. Ono, "Red ginseng ameliorated place navigation deficits in young rats with hippocampal lesions and aged rats," Physiology \& Behavior, vol. 69, no. 4-5, pp. 511-525, 2000.

[57] L. Li, J. Liu, X. Yan et al., "Protective effects of ginsenoside Rd against okadaic acid-induced neurotoxicity in vivo and in vitro," Journal of Ethnopharmacology, vol. 138, no. 1, pp. 135-141, 2011.

[58] Y. Wang, J. Liu, Z. Zhang, P. Bi, Z. Qi, and C. Zhang, "Antineuroinflammation effect of ginsenoside $\mathrm{Rbl}$ in a rat model of Alzheimer disease," Neuroscience Letters, vol. 487, no. 1, pp. 70-72, 2011.

[59] T. Huang, F. Fang, L. Chen et al., "Ginsenoside Rg1 attenuates oligomeric A\&beta;1-42-Induced mitochondrial dysfunction," Current Alzheimer Research, vol. 9, no. 3, pp. 388-395, 2012.

[60] J. S. Zhou, J. F. Wang, B. R. He et al., "Ginsenoside Rd attenuates mitochondrial permeability transition and cytochrome $\mathrm{C}$ release in isolated spinal cord mitochondria: involvement of kinase-mediated pathways," International Journal of Molecular Sciences, vol. 15, no. 6, pp. 9859-9877, 2014. 
[61] M. Sun, C. Huang, C. Wang et al., "Ginsenoside Rg3 improves cardiac mitochondrial population quality: mimetic exercise training," Biochemical and Biophysical Research Communications, vol. 441, no. 1, pp. 169-174, 2013.

[62] H. Lee, Y. Hong, Q. Tran et al., “A new role for the ginsenoside RG3 in antiaging via mitochondria function in ultravioletirradiated human dermal fibroblasts," Journal of Ginseng Research, vol. 43, 2018.

[63] X. Yan, G. Hu, W. Yan et al., "Ginsenoside Rd promotes nonamyloidogenic pathway of amyloid precursor protein processing by regulating phosphorylation of estrogen receptor alpha," Life Sciences, vol. 168, pp. 16-23, 2017.

[64] R. J. Choi, A. Roy, H. J. Jung et al., "BACE1 molecular docking and anti-Alzheimer's disease activities of ginsenosides," Journal of Ethnopharmacology, vol. 190, pp. 219-230, 2016.

[65] F. Fang, X. Chen, T. Huang, L. F. Lue, J. S. Luddy, and S. S. Yan, "Multi-faced neuroprotective effects of ginsenoside Rg1 in an Alzheimer mouse model," Biochimica et Biophysica Acta, vol. 1822, no. 2, pp. 286-292, 2012.

[66] G. Cao, P. Su, S. Zhang et al., "Ginsenoside Re reduces A $\beta$ production by activating PPAR $\gamma$ to inhibit BACE1 in N2a/APP695 cells," European Journal of Pharmacology, vol. 793, pp. 101-108, 2016.

[67] Z. Y. Mo, Y. Z. Zhu, H. L. Zhu, J. B. Fan, J. Chen, and Y. Liang, "Low micromolar zinc accelerates the fibrillization of human tau via bridging of Cys-291 and Cys-322," The Journal of Biological Chemistry, vol. 284, no. 50, pp. 34648-34657, 2009.

[68] S. Barghorn and E. Mandelkow, "Toward a unified scheme for the aggregation of tau into Alzheimer paired helical filaments," Biochemistry, vol. 41, no. 50, pp. 14885-14896, 2002.

[69] M. E. King, T. C. Gamblin, J. Kuret, and L. I. Binder, "Differential assembly of human tau isoforms in the presence of arachidonic acid," Journal of Neurochemistry, vol. 74, no. 4, pp. 1749-1757, 2000.

[70] T. Kampers, P. Friedhoff, J. Biernat, E. M. Mandelkow, and E. Mandelkow, "RNA stimulates aggregation of microtubuleassociated protein tau into Alzheimer-like paired helical filaments," FEBS Letters, vol. 399, no. 3, pp. 344-349, 1996.

[71] M. Goedert, R. Jakes, M. G. Spillantini, M. Hasegawa, M. J. Smith, and R. A. Crowther, "Assembly of microtubuleassociated protein tau into Alzheimer-like filaments induced by sulphated glycosaminoglycans," Nature, vol. 383, no. 6600, pp. 550-553, 1996.

[72] E. E. Congdon and K. E. Duff, "Is tau aggregation toxic or protective?," Journal of Alzheimer's Disease, vol. 14, no. 4, pp. 453457, 2008.

[73] K. Santacruz, J. Lewis, T. Spires et al., "Tau suppression in a neurodegenerative mouse model improves memory function," Science, vol. 309, no. 5733, pp. 476-481, 2005.

[74] R. H. Ma, Y. Zhang, X. Y. Hong, J. F. Zhang, J. Z. Wang, and G. P. Liu, "Role of microtubule-associated protein tau phosphorylation in Alzheimer's disease," Journal of Huazhong University of Science and Technology. Medical Sciences, vol. 37, no. 3, pp. 307-312, 2017.

[75] K. Iqbal, A. . C. Alonso, and I. Grundke-Iqbal, "Cytosolic abnormally hyperphosphorylated tau but not paired helical filaments sequester normal MAPs and inhibit microtubule assembly," Journal of Alzheimer's Disease, vol. 14, no. 4, pp. 365-370, 2008.

[76] A. D. Alonso, I. Grundke-Iqbal, H. S. Barra, and K. Iqbal, "Abnormal phosphorylation of tau and the mechanism of
Alzheimer neurofibrillary degeneration: sequestration of microtubule-associated proteins 1 and 2 and the disassembly of microtubules by the abnormal tau," Proceedings of the National Academy of Sciences of the United States of America, vol. 94, no. 1, pp. 298-303, 1997.

[77] A. S. Chesser, S. M. Pritchard, and G. V. W. Johnson, “Tau clearance mechanisms and their possible role in the pathogenesis of Alzheimer disease," Frontiers in Neurology, vol. 4, 2013.

[78] M. J. Lee, J. H. Lee, and D. C. Rubinsztein, “Tau degradation: the ubiquitin-proteasome system versus the autophagylysosome system," Progress in Neurobiology, vol. 105, pp. 4959, 2013.

[79] Y. Wang and E. Mandelkow, "Degradation of tau protein by autophagy and proteasomal pathways," Biochemical Society Transactions, vol. 40, no. 4, pp. 644-652, 2012.

[80] A. Mudher, M. Colin, S. Dujardin et al., "What is the evidence that tau pathology spreads through prion-like propagation?," Acta Neuropathologica Communications, vol. 5, no. 1, p. 99, 2017.

[81] C. E. G. Leyns and D. M. Holtzman, "Glial contributions to neurodegeneration in tauopathies," Molecular Neurodegeneration, vol. 12, no. 1, p. 50, 2017. 